\title{
Concept of a nanowire array magnetoresistance device
}

\author{
I. V. Shvets, ${ }^{\text {a) }}$ H. C. Wu, V. Usov, F. Cuccureddu, S. K. Arora, and S. Murphy \\ CRANN, School of Physics, Trinity College, Dublin 2, Ireland
}

(Received 2 October 2007; accepted 20 December 2007; published online 15 January 2008)

\begin{abstract}
We describe a concept of the magnetoresistive medium comprising of a planar array of intermingled nanowires with pinned and unpinned magnetic moments. We propose a bottom up method of forming the medium and demonstrate the feasibility of the proposed approach. We present the results of the simulations of the magnetic moments in the nanowires under an external magnetic field to illustrate the concept. (C) 2008 American Institute of Physics. [DOI: 10.1063/1.2834371]
\end{abstract}

Typically a magnetoresistive element consists of separated magnetic pinned (PL) and free layers (FL). In these nanostructures, the magnetoresistive effect originates mainly from spin-dependent scattering at the interface between a PL/FL and a nonmagnetic spacer. ${ }^{1}$ Alternatively, in magnetic tunneling junctions (MTJs), spin dependent tunneling through an insulating spacer leads to a large spin asymmetry and correspondingly to magnetoresistance. ${ }^{2}$ Such giant magnetoresistance (GMR) and tunneling magnetoresistance (TMR) nanoelements are fabricated using a top down lithography-based process, leading to an out-of-plane oriented array of devices commonly operated in the current perpendicular to the plane mode.

Recently, increasing attention has been paid to an alternative bulklike mechanism of spin dependent scattering due to domain walls (DWs) in magnetic nanowires. ${ }^{3,4}$ In bulk structures, this mechanism leads to a relatively small magnetoresistive (MR) effect. This small effect is due to a strong dependence of MR on the DW thickness. This dependence has been investigated in detail theoretically, ${ }^{5-10}$ showing that a very narrow DW leads to MR values on the order of $100 \%$. Since DW thickness can be significantly reduced in narrow nanowires, nanoconstrictions, and nanocontacts, significant efforts have been recently devoted to the fabrication and characterization of such nanostructures. Typically, such structures are also fabricated using top-down lithographybased nanofabrication techniques. For the present discussion, it is important to note that, overall, the DW scattering mechanism is expected to lead to an order of magnitude smaller MR effect than spin dependent scattering in MTJs or GMR structures.

Here, we propose the concept of a magnetoresistive element and fabrication approach which is attractive due to its relative simplicity, as it does not involve multiple lithography steps. Instead, we use the atomic terrace low angle shadowing (ATLAS) technique, which has the advantage of bottom-up fabrication. We discuss the concept of the MR nanostructure, which utilizes DW scattering where the MR is further enhanced by creating multiple DW's in a nanowire array traversed by the in-plane spin-polarized electron current. Contrary to a conventional approach of single nanowires, we propose using dense array of magnetic wires to stabilize the DW network.

The proposed medium consists of a planar array of nanowires with a width of some $5-50 \mathrm{~nm}$ (Fig. 1). Two types of nanowires are used, the so-called spacer nanowires, marked

\footnotetext{
${ }^{\text {a) }}$ Author to whom correspondence should be addressed. Electronic mail:
} ivchvets@tcd.ie. hatched, and magnetoresistive nanowires composed of, e.g., $\mathrm{Fe}$ or Co, marked gray. The substrate is an antiferromagnetic single crystal, e.g., NiO. The spacer nanowires are of an insulating or semiconductor material that destroys exchange coupling between the substrate and the magnetoresistive nanowire. Thus, two subsets of magnetoresistive nanowires are formed: the nanowires positioned on the bare substrate and the ones positioned on top of the spacer nanowires. These are shown in Figs. 1(a) and 1(b) in the light gray and dark gray colors, respectively. Since the magnetoresistive nanowires of the first subset are exchange coupled to an antiferromagnetic substrate, the field that is required to rotate magnetisation in these wires is different from that of the magnetoresistive nanowires of the second subset, which are decoupled from the substrate by an array of spacer nanowires. Therefore, as the magnetic field is applied, it should be possible to switch between the two configurations in which the magnetoresistive nanowires of the two subsets are magnetized antiparallel or parallel to each other [Figs. 1(a) and 1(b), respectively]. This medium is expected to have a large magnetoresistance, with current passing in plane, in the direction perpendicular to the nanowires. The rationale is that the domain wall between nanowires is expected to be narrow. Below we present the model to substantiate this point. Furthermore, the material could ultimately contain a high number of the domain walls in the path of the current, thus providing a large signal during measurements.

The required array of nanowires could be formed on vicinal surface by means of the ATLAS technique. Vicinal surfaces can be formed by annealing a surface that is off cut from a low-index orientation. It is composed of atomic terraces separated by steps. The width of the atomic terraces can be readily controlled by the off-cut angle. Therefore, the separation between the nanowires within the array and their width could be controlled.

In the proposed ATLAS technique, a collimated beam of evaporant atoms is directed at the vicinal surface at a shallow angle $\beta$ of only $1^{\circ}-3^{\circ}$ [Fig. 1(c)]. Some areas of the atomic terraces are exposed to the beam while others are shadowed. The flux direction in relation to the step edges determines what areas of the atomic terrace are exposed or shadowed. The width of the nanowires is defined by

$$
w=a\left(\frac{1}{\alpha}-\frac{1}{\alpha+\beta}\right),
$$

where $w$ is a nanowire width, $a$ is a step height, $\alpha$ is a miscut angle, and $\beta$ is a deposition angle, as shown in Fig. 1(c). Both $\alpha$ and $\beta$ are assumed to be small. 


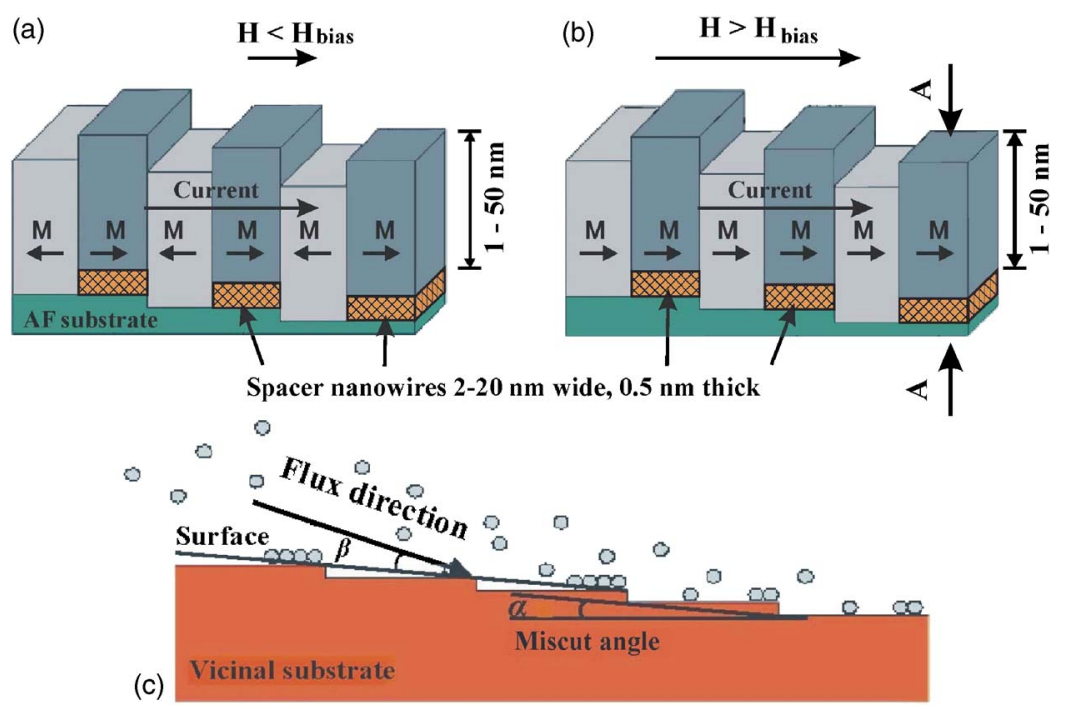

FIG. 1. (Color online) Schematics of the MR medium showing the magnetization of the nanowires for (a) an applied magnetic field (a) smaller and (b) larger than an exchange bias field (c) schematics of deposition of nanowires on the outer step edges of a vicinal surface; the beam is directed at a shallow angle $\beta$ along the descending step direction.

The proposed spacer nanowires are to be deposited by the ATLAS process and the magnetoresistive layer is to be deposited by means of conventional deposition techniques. Figure 1(c) shows the beam directed at the substrate along the descending step direction.

We demonstrated the feasibility of forming arrays of nanowires down to $10-30 \mathrm{~nm}$ width by ATLAS using two types of substrates: $\mathrm{Si}(111)$ and $c$-plane (0001) sapphire $\left(a-\mathrm{Al}_{2} \mathrm{O}_{3}\right)$.

Silver nanowires were grown on $\mathrm{Si}(111)$ step-bunched surface. The substrates were prepared from $\operatorname{Si}(111) n$-type doped wafer with a $3.2^{\circ}$ miscut along the $[-1-12]$ direction. To obtain a step-bunched $\mathrm{Si}(111)$ surface the samples were annealed by dc current. The obtained surface morphology is characterized by a staircase arrangement of terraces, which are $25-30 \mathrm{~nm}$ wide and separated by bunches of steps with a height of $2.5 \pm 1 \mathrm{~nm}$ [Fig. 2(a)].
A collimated Ag flux from an effusion cell was oriented perpendicular to the direction of the step edges in the descending step direction at $1^{\circ}-2^{\circ}$ to the sample surface. Figures 2(b) and 2(c) show the silver nanowires deposited at $\beta=1.2^{\circ}$ deposition angle. The wires' average height, width, and separation are 2,15 , and $30 \mathrm{~nm}$, respectively. It is interesting that the height of the nanowires, some $2 \mathrm{~nm}$, is well in excess of a single atomic layer. The width of $15 \mathrm{~nm}$ is approximately a fivefold reduction in size compared to existing benchmark results for nanowire arrays produced by deposition at shallow angle. ${ }^{11}$

In another series of experiments, we used the substrate of $\alpha-\mathrm{Al}_{2} \mathrm{O}_{3} c$-plane (0001) sapphire with a $3^{\circ}$ miscut along the (1-210) direction. To form the atomic terraces, the substrate was annealed in the air at $1100{ }^{\circ} \mathrm{C}$. A flux of collimated Co atoms was used. Figure 2(d) shows the represen-
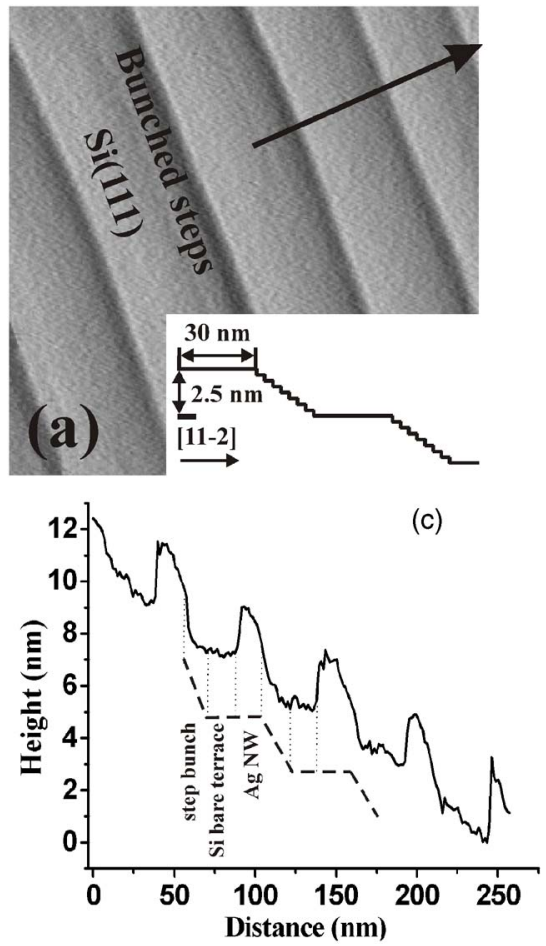
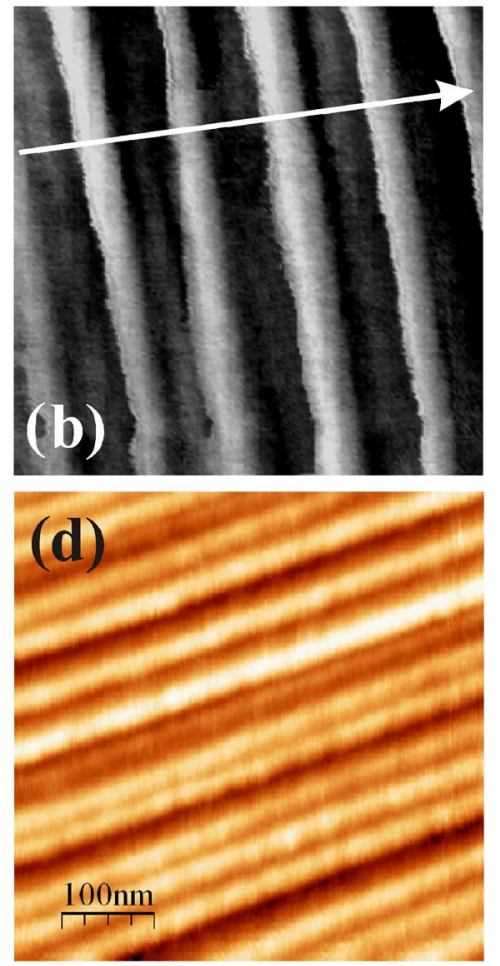

FIG. 2. (Color online) (a) $135 \times 135 \mathrm{~nm}^{2}$ derivative AFM image of an array of bunched steps on a miscut $\mathrm{Si}(111)$ surface. Light stripes are flat $\mathrm{Si}(111)$ terraces while the darker regions correspond to the bunched steps; the inset is a schematic along the direction of a miscut marked in (a) by an arrow. (b) $250 \times 250 \mathrm{~nm}^{2}$ AFM image of a planar array of $\mathrm{Ag}$ nanowires grown at $\beta=1.2^{\circ}$. (c) A line profile along the direction of a miscut marked in (b) and a schematic of an underlying $\mathrm{Si}(111)$ bunched substrate. (d) AFM image of Co nanowires deposited on miscut $c$-plane $\alpha-\mathrm{Al}_{2} \mathrm{O}_{3}$. 

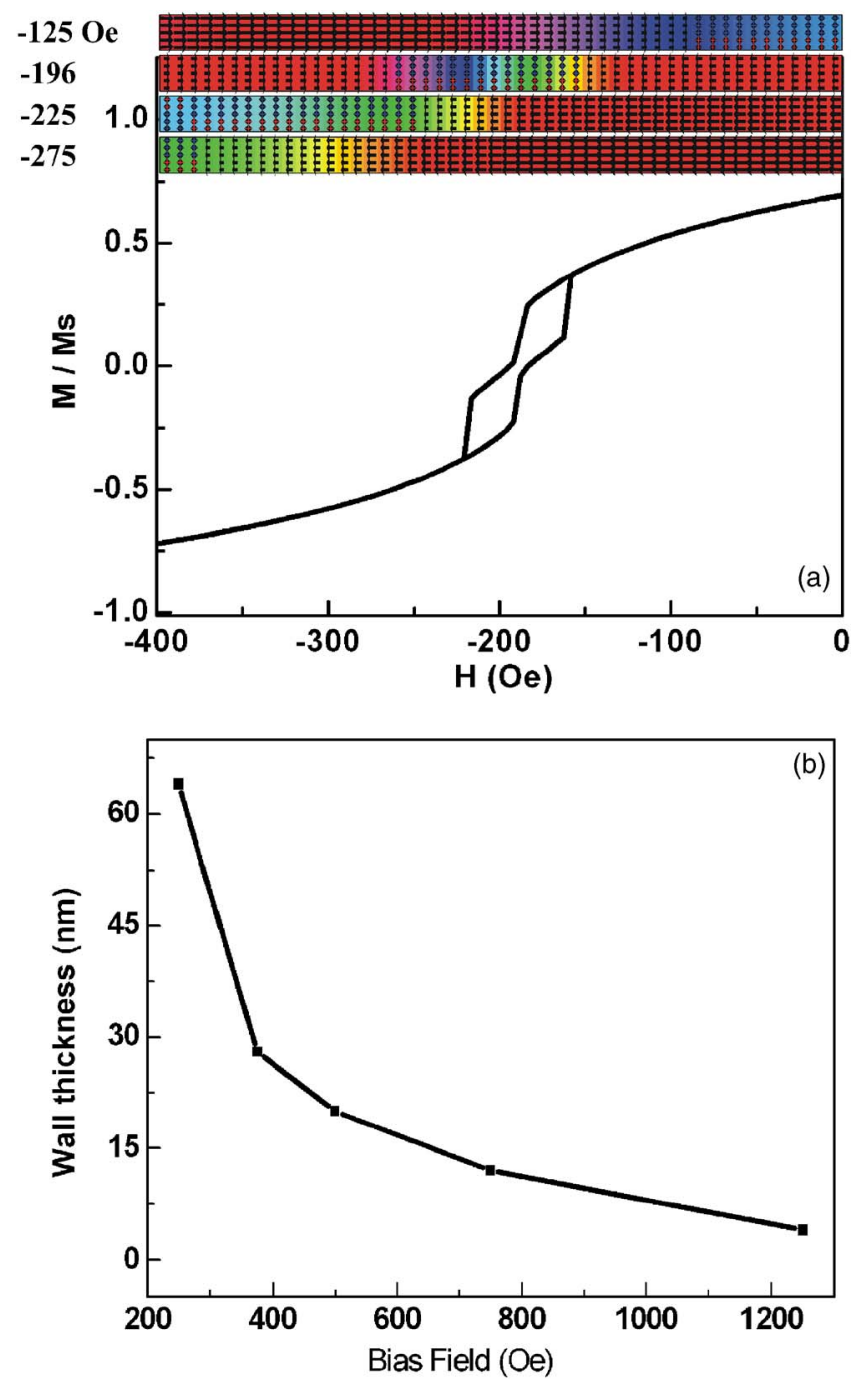

FIG. 3. (Color online) (a) Hysteresis loop simulation for a Ni film separated into two nanowires $(250 \times 50 \times 5 \mathrm{~nm})$. The insets are the vector maps for different external fields; the cross section is along $A-A$ plane [Fig. 1(a)] perpendicular to the nanowire length. (b) Simulated domain wall width as a function of bias field for a domain wall between neighboring nanowires having pinned and unpinned magnetic moments. A bias field applied to the pinned nanowire is $375 \mathrm{Oe}$.

tative area of the sample. The nanowires formed in this experiment had the width of $17 \mathrm{~nm}$.

We simulated the formation of a domain wall in the structure as described in the letter using micromagnetic calculation (OOMMF code). ${ }^{12}$ For the sake of simplification, we consider a single MR unit cell comprising of two nanowires, one pinned nanowire and one unpinned nanowire ferromagnetically coupled to each other. The dimensions of each of the nanowires are $250 \times 50 \times 5 \mathrm{~nm}$. The strength of antiferromagnetic coupling between the pinned nanowire and the antiferromagnetic material was introduced by a bias field which was adjusted in the range from 250 to 1250 Oe. Four energies are considered: exchange energy, anisotropy energy, magnetostatic energy, and Zeeman energy. We used the following parameters for $\mathrm{Ni}: M_{s}=493 \times 10^{3} \mathrm{~A} / \mathrm{m}, \quad \mathrm{A}=7.2$ $\times 10^{-12} \mathrm{~J} / \mathrm{m}$, cubic anisotropy constants $K_{1}=-4.5$ $\times 10^{3} \mathrm{~J} / \mathrm{m}^{3}, \quad K_{2}=-2.5 \times 10^{3} \mathrm{~J} / \mathrm{m}^{3}$, and a large artificial damping coefficient 0.5 . Figure $3(\mathrm{a})$ shows the results of a calculation for the case of the bias field applied to the pinned $\mathrm{Ni}$ nanowire of 375 Oe. Figure 3(b) shows the numerically simulated magnetic domain wall thickness as a function of the exchange bias field. The domain walls for strongly pinned nanowires are much narrower than the Bloch walls for Ni. The domain wall as narrow as $5-7 \mathrm{~nm}$ [Fig. 3(b)] should correspond to the estimated MR value of $15-100 \% .^{13}$ The width of the domain wall could be further reduced due to the breakdown in the exchange interaction between the neighboring nanowires resulting from the structural defects that are known to exist along the atomic terrace step edge in ultrathin films.

We proposed the concept of a magnetoresistive medium utilising a planar array of ferromagnetic nanowires. The medium is based on pinning the magnetic moment of every second nanowire in the array and thus engineering the width of the domain wall. We proposed the method of fabrication of the medium, utilising a vicinal substrate, and demonstrated its feasibility.

The authors would like to thank O. N. Mryasov from Research Seagate Technology, Pittsburg and Ted Kamins from Hewlett-Packard Laboratories, Palo-Alto for helpful discussions. The financial support of Science Foundation Ireland, Contract No. 06-IN.1/I91 is gratefully acknowledged.

${ }^{1}$ E. Y. Tsymbal and D. G. Pettifor, Solid State Physics, edited by H. Ehrenreich and F. Spaepen (Academic, New York, 2001), Vol. 56, pp. 113-237, and references therein.

${ }^{2}$ E. Y. Tsymbal, O. N. Mryasov, and P. R. LeClair, J. Phys.: Condens. Matter 15, R109 (2003), and references therein.

${ }^{3}$ A. D. Kent, U. Rudiger, J. Yu, L. Thomas, and S. S. P. Parkin, J. Appl. Phys. 85, 5243 (1999).

${ }^{4}$ C. L. Dennis, R. P. Borges, L. D. Buda, U. Ebels, J. F. Gregg, M. Hehn, E. Jouguelet, K. Ounadjela, I. Petej, I. L. Prejbeanu, and M. J. Thornton, J. Phys.: Condens. Matter 14, R1175 (2002).

${ }^{5}$ H. Imamura, N. Kobayashi, S. Takahashi, and S. Maekawa, Phys. Rev. Lett. 84, 1003 (2000).

${ }^{6}$ L. R. Tagirov, B. P. Vodopyanov, and K. B. Efetov, Phys. Rev. B 65, 214419 (2002).

${ }^{7}$ V. K. Dugaev, J. Berakdar, and J. Barnas, Phys. Rev. B 68, 104434 (2003).

${ }^{8}$ J. J. Mallett, E. B. Svedburg, H. Ettedaui, T. P. Moffat, and W. F. Egelhoff, Jr., Phys. Rev. B 70, 172406 (2004).

${ }^{9}$ R. F. Sabirianov, A. K. Solanki, J. D. Burton, S. S. Jaswal, and E. Y. Tsymbal, Phys. Rev. B 72, 054443 (2005).

${ }^{10}$ P. E. Falloon, R. A. Jalabert, D. Weinmann, and R. Stamps, Phys. Rev. B 70, 174424 (2004).

${ }^{11}$ J. Oster, M. Kallmayer, L. Wiehl, H. J. Elmers, H. Adrian, F. Porrati, and M. Huth, J. Appl. Phys. 97, 014303 (2005).

${ }^{12}$ M. J. Donahue and D. G. Porter, oOMmF user's guide, version 1.2a. Technical Report NISTIR 6376, National Institute of Standards and Technology, Gaithersburg, MD, 1999, URL: (http://math.nist.gov/oommf).

${ }^{13}$ P. M. Levy and S. Zhang, Phys. Rev. Lett. 79, 5110 (1997). 